de l'intérêt de cette initiative. Lorsqu'un patient se trouve dans une situation d'errance de diagnostic, il peut publier son cas directement, de manière anonyme, et le mettre à disposition de l'ensemble de la communauté médicale. L'aspect éthique doit être pris en compte dans ce type de démarche.

\section{Les nouveaux outils}

S'agissant des essais cliniques, une étude présentée à Boston a démontré que les propositions et récoltes d'information par Facebook étaient plus pertinentes que d'autres, s'agissant du nombre de volontaires inclus.

Par ailleurs, les serious games peuvent permettre à des enfants de mieux comprendre leur maladie et à leurs parents de mieux les accompagner. Ce type de jeux peut également donner des outils aux enseignants, dans le cadre d'une approche pédagogique.

En cas d'errance diagnostique, un accompagnement est nécessaire par la simulation numérique. Il est ainsi possible, par exemple, de prendre en charge un patient atteint d'accidents vasculaires cérébraux dans toutes les étapes. Le raisonnement clinique peut permettre de comprendre comment le patient doit être accompagné de son domicile à l'hôpital et en centre de rééducation.

IBM s'implique par exemple fortement dans l'intelligence artificielle. Il est ainsi possible de disposer d'un recueil d'informations consultables dans des bases extrêmement importantes et internationales, qui ne pourrait jamais être constitué par un médecin. Aujourd'hui, ce type d'outil doit encore s'améliorer, mais peut aider le médecin à prendre une décision plus rapidement et à passer plus de temps avec le patient.

Les Natou sont Netflix, Airbnb, Telsa Motors et Uber. Uber permet de se déplacer dans de nombreuses villes du monde, mais propose parfois d'autres solutions. En Inde, Uber $X$ permet de livrer des médicaments à domicile.

La e-santé ne doit pas s'enfermer dans une démarche trop technologique et réglementaire, mais favoriser l'échange entre les patients et les professionnels et apporter des solutions utiles. Les acteurs numériques, souvent américains, ont trouvé des solutions. De nombreux acteurs du monde hospitalier et de la recherche souhaitent par ailleurs pouvoir proposer ce type de solutions.

Pour conclure, un élément me semble essentiel. Le cœur de la e-santé, ce sont les lieux de rencontre entre médecins, développeurs, ingénieurs, pharmaciens et patients qui vont imaginer la santé de demain. L'innovation de demain en santé se conçoit avant tout avec les patients et les professionnels de santé. $\diamond$

State of play of e-health technologies

\section{LIENS D'INTÉRÊT}

L'auteur déclare n'avoir aucun lien d'intérêt concernant les données publiées dans cet article.

\title{
État des lieux des expérimentations innovantes en télémédecine en France
}

\section{Bilan 2018 : une évolution progressive vers la maturité du déploiement technologique}

Mon expérience est celle d'un praticien interniste, urgentiste, qui travaille dans la télémédecine (TLM) depuis 2004. J'ai acquis un regard à travers ce qui se passe dans différentes régions, chaque région étant organisée en silo. Les silos entre spécialités et la médecine de ville se sont reproduits dans les systèmes d'information et de communication. Comment faire pour dépasser cela?

La première étape est de disposer de l'outil de visioconférence. Développer des réunions de concertation pluridisciplinaires entre centres de

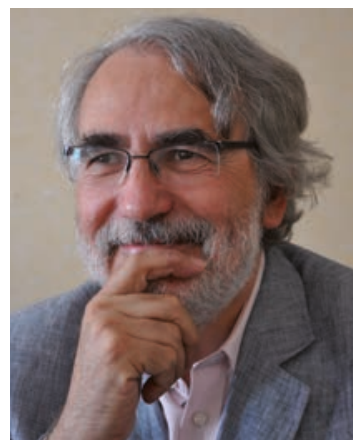

Consultant espinoza.telemedecine@ gmail.com www.sf-telemed.org

référence, centres de compétences et médecins traitants est un objectif prioritaire. Ces réunions favoriseront les transferts de savoir et permettront de conforter une rela- 
1 Bilan des textes et de la règlementation légale pour les usages de la TLM et les technologies (messagerie sécurisée, objets connectés, dispositifs médicaux)

1. La loi HPST en août 2004 reconnaît la TLM comme de la médecine à distance

2. Le décret de TLM du 19/10/2010 identifie 5 actes de TLM

3. La circulaire de la DGOS a identifié en 2012 cinq priorités nationales pour la TLM

4. La loi de FSS 2018 propose une tarification des actes de TLM dans le droit commun

5. Les circulaires DGOS ANAP ASIP HAS proposent des recommandations pour la TLM*

6. Le $\mathrm{CNOM}^{*}$ propose des recommandations pour la TLM en 2009 et en 2013 pour les objets connectés

7. Les dispositifs médicaux, les objets connectés, l'intelligence artificielle devraient s'intégrer dans les organisations

* Documents et textes de loi sont accessibles sur les sites internet Gouv HAS ASIP CNOM.

tion de confiance. L'usage de la visioconférence est également un élément clé pour renforcer la formation mais aussi les liens entre les centres de recherche notamment avec leurs partenaires centres de compétence, sans oublier le médecin traitant qui ne doit pas être exclu.

Quelle est la configuration technologique nécessaire? Les progrès dans ce domaine ont été considérables. II existe différents types de stations de visioconférences. La station classique utilise un codec, c'est-à-dire «un ordinateur spécialisé » qui transporte le son, l'image, partage des comptes rendus voir des clichés radiologiques. Communiquer entre centres de référence, centres de compétences et filières implique de concevoir puis financer un plan de déploiement centré sur la bonne configuration technologique à mettre en place et l'accompagnement métier. Aujourd'hui des technologies de visioconférence utilisant, des tablettes, smartphones, des ordinateurs standards sont disponibles avec intégration de la visioconférence et le partage de documents.

Pour le déploiement il faut construire un projet en confiance avec les départements informatiques des hôpitaux, il est possible d'équiper les ordinateurs de webcams et de systèmes audio en veillant naturellement à la sécurisation et confidentialité des données. Ces besoins techniques nécessitent un financement et un accompagnement métier pour favoriser leurs usages. N'oublions pas les contraintes en termes de réseau dont l'impact économique, l'accessibilité sont variables selon la structure et le territoire.

\section{Une simplification des textes s'impose}

Les textes réglementaires (Encadré I) reconnaissent la télémédecine comme de la médecine à distance, la responsabilité des professionnels obéit aux mêmes règles que pour la médecine. Le décret de TLM a identifié cinq actes de télémédecine. La DGOS a préconisé cinq priorités nationales, qui se déclinent ensuite dans les programmes régionaux de télémédecine, avec la construction de plateformes régionales qui fonctionnent en silo. Parmi les textes les recommandations HAS du guide de pilotage et de sécurité de télémédecine de 2013 ont défini une stratégie qui devrait être réactualisée ainsi que le décret de TLM de 2010. La HAS a publié son guide formalisant la procédure: «le projet médical doit être défini avant le choix d'un outil technologique », la stratégie de déploiement doit organiser la conception, l'installation, la mise en route, le fonctionnement régulier et le retour d'expérience. ARS et GCS, bientôt GIP pilotent la gouvernance régionale de chaque solution régionale.

L'un des points clefs du déploiement de la télémédecine est le financement et la tarification des actes. Pendant des années, nous avons été confrontés à ce serpent de mer. En 2011, une loi de financement prévoyait la tarification, mais l'assurance maladie n'a pas provisionné de budget pour financer ces actes. Des décrets ont été publiés ensuite sur les plaies, ils constituaient, toutefois, de véritables usines à gaz administratives non applicables par les professionnels du terrain. En 2017, il était prévu de procéder à une expérimentation concernant les tarifications (Projet ETAPE).

La loi de financement de la sécurité sociale 2018 vise, enfin, à promouvoir la tarification des actes de TLM dans le droit commun mais des décrets devront être publiés. Un espace nouveau devrait donc s'ouvrir. Pour mettre en place cette tarification expérimentale, il faudra, bien sûr, négocier les modalités conventionnelles avec l'assurance maladie.

\section{Le besoin des filières maladies rares}

L'Encadré 2 définit les actes du décret de 2010 conditionnant la tarification opérationnelle. L'étude des besoins dans une filière de maladie rare sur un territoire inter-régional devrait identifier les usages potentiels des actes de TLM définis par la loi d’octobre 2010. La téléexpertise doit pouvoir être effectuée dans un cadre juridique et technologique sécurisé, elle facilite le tri et permet une première évaluation. La téléconsultation doit éviter de déplacer des patients fragiles et assurer un suivi pertinent. La télésurveillance peut porter sur des pathologies pour faciliter le transfert de savoir. La téléassistance vise par exemple à assister un patient pour faire un pansement, un geste technique. Dans le cadre des maladies rares, de nombreux actes de télémédecine peuvent être réalisés, qui apporteraient un bénéfice aux patients, aux partenaires et à l'organisation des soins. Les procédures organisationnelles à négocier entre les partenaires sont des points clés du succès d'un déploiement. 


\section{Propositions pour développer l'usage des technologies} dans les maladies rares

1. Réaliser une évaluation des besoins sur une filière associant CRMR, CCMR, généralistes

2. Développer l'accès à la visioconférence, la messagerie sécurisée territoriale entre les partenaires

3. La visioconférence permet des RCP, la formation et le suivi de la recherche

4. Définir un projet médical centré sur les besoins, identifier l'impact technico-fonctionnel

5. Veiller à la cohérence technique et organisationnelle entre les partenaires

6. La modélisation sur une filière doit servir pour la généralisation des outils, de l'organisation

Le bilan des priorités 2012 de la DGOS montre :

- Le déploiement de la téléradiologie requiert parfois des technologies et partenariats industriels différents d'une région à l'autre.

- Le déploiement pour l'AVC a été significatif dans de nombreuses régions.

- L'activité de TLM a été faible pour les soins aux détenus alors que les besoins sont majeurs.

- Le bilan de la TLM est mitigé pour les maladies chroniques et les patients en HAD.

- Un besoin bien ciblé pour les EHPAD et les MAS.

Dans tous les projets de TLM qui ont été reconnus avec une activité significative la coopération entre les professionnels de santé reconnue par l'article 51 de la loi HPST est un élément clef du déploiement qui conditionne l'appropriation de la TLM par les professionnels.

L'Encadré 3 suggère, pour les maladies rares, parmi les propositions de choisir une filière maladie rare, de définir dans cette filière pilote le projet médical des TIC et de la TLM, de développer l'usage de la visioconférence entre les centres de références et les centres de compétences, de systématiser les réunions de concertation pluridisciplinaires, d'organiser des formations à distance et de définir un projet médical centré sur les besoins dans la filière. Un véritable plan de déploiement national des TIC (technologies d'information et de communication) et de la TLM est indispensable, sans oublier les objets connectés et les dispositifs médicaux dont la sélection, la validation devront être qualifiées par les professionnels. La modélisation sur un périmètre limité doit fournir des arguments concrets pour réussir un déploiement plus large et cohérent selon des critères d'efficience organisationnelle et médico-économique.

\section{Le décret d'octobre 2010 définit 5 actes de TLM}

Téléconsultation : un médecin donne une consultation à distance à un patient, un professionnel de santé peut être présent auprès du patient et, le cas échéant, assister le professionnel médical au cours de la téléconsultation TLC. Les psychologues peuvent également être présents près du patient.

Téléexpertise : un médecin sollicite à distance en TLX l'avis d'un ou plusieurs médecins en raison de leurs formations ou compétences particulières, sur la base des informations médicales.

Télésurveillance : a pour objet de permettre à un professionnel médical d'interpréter à distance les données nécessaires au suivi médical d'un patient et, le cas échéant, de prendre des décisions relatives à la prise en charge du patient. L'enregistrement et la transmission des données peuvent être automatisés ou réalisés par le patient lui-même ou par un professionnel de santé.

Téléassistance : a pour objet de permettre à un professionnel médical d'assister à distance un autre professionnel de santé au cours de la réalisation d'un acte. Régulation médicale : cible la réponse médicale apportée par les centres 15 .

Pour réussir il faut « démarrer petit » sur une filière pilote avec des professionnels impliqués et motivés, modéliser les usages selon l'activité médicale, les besoins des patients. L'accompagnement métier est un point clé qui nécessite que la gouvernance du projet prenne en compte tous les facteurs clés du déploiement, non seulement la tarification, mais tous les freins connus, identifiés sur le terrain. Le succès passe aussi par la nécessité de construire un projet cohérent comme l'a souligné le rapport de la cour des comptes de novembre 2017. Le financement d'un «plan maladies rares et déploiement technologique de la e-santé » devrait s'imposer pour faciliter l'accès aux soins et le soutien de professionnels souvent isolés à distance des centres de référence. $\diamond$ State of play of innovative experimentations in telemedicine

\section{LIENS D'INTÉRÊT}

L'auteur déclare n'avoir aucun lien d'intérêt concernant les données publiées dans cet article. 